\title{
猪诱导性多能干细胞技术的研究进展及应用前景
}

\author{
间益波，张艳丽，齐巍巍，万永杰，牀懿荁，王锋
}

南京农业大学动物胚胎工程技术中心，南京 210095

摘要: 猪作为实验材料, 具有由于来源方便、基因序列与人类的相近及其在畜牧业中的重要地位等优势, 成为国内外研究的热点, 但是猪的肧胎干细胞 (Embryonic stem cells, ESC) 建系方面的研究进展缓慢。诱导性多 能干细胞 (induced pluripotent stem cells, iPSC)技术的诞生, 开创了体细胞重编程的全新方法。猪 iPSC 体系的建 立将为家畜 $\mathrm{ESC}$ 体系的建立奠定基础, 同时也对提高猪转基因克隆的效率, 高效育种和保种, 乃至生物医学领 域均产生深远的影响。文章综述了 iPSC 技术的主要进展, 重点阐述了猪 iPSC 技术的现状及其在生物医学和畜 牧业中的应用前景, 以期为从事该领域研究的科研人员提供参考。

关键词：猪; 诱导性多能干细胞; 胚胎干细胞; 应用前景

\section{Progress and application prospect of pig induced pluripotent stem cells}

\author{
YAN Yi-Bo, ZHANG Yan-Li, QI Wei-Wei, WAN Yong-Jie, FAN Yi-Xuan, WANG Feng \\ Center of Animal Embryo Engineering and Technology, Nanjing Agricultural University, Nanjing 210095, China
}

\begin{abstract}
Pig has always been the focus of establishing a big ungulate animal ES cell lines because of its convenient source, genetic similarity with humans, and their importance in animal husbandry, but little development is achieved. Induced pluripotent stem cells technology creates a new method of reprogramming somatic cells to pluripotent state. As the pig iPS cells is established and perfected, pig ES cells will be established in the coming years. The pig iPS cells will give a hint on other livestock ES cells. On the other hand, pig iPS cells can be used to improve the efficiency of transgenic cloning pigs to conduct effective breeding and conservation of breeds. It is particularly important that the pig iPS cells can provide new model for human medical research, a new donor cells for human tissue and organ engineering, and have extensive and far-reaching impact on the biomedical field. Here, we briefly review the major progress of iPS cells, and emphasize current state of pig iPS cells and its application prospect in biomedicine and animal husbandry in order to provide a useful reference for researchers working in this area.
\end{abstract}

Keywords: pig; induced pluripotent stem cells; embryonic stem cells; application prospect

胚胎干细胞(Embryonic stem cells, ESC)是从早 期胚胎的囊胚内细胞团细胞(Inner cell mass, ICM)或 胎儿原始生殖细胞(Primordial germ cell, PGC)经分 离、体外抑制分化培养得到的具有发育全能性的细
胞。 $\mathrm{ESC}$ 的分离培养建系是胚胎生物技术领域的重 大成就，同时也是现代生物技术研究的热点和焦点, 具有非常重要的研究价值。基础方面主要应用于研 究基因功能、细胞分化和个体发育; 应用方面主要

收稿日期: 2010-08-02; 修回日期: 2010-09-29

基金项目: 国家高技术研究发展计划(863)项目(编号：2008AA101005)和国家自然科学基金项目(编号 : 30771554)资助

作者简介: 问益波, 博士研究生, 研究方向：胚胎干细胞。Tel: 15996276236; E-mail: bobo10679888@163.com

通讯作者: 王锋, 博士, 教授, 研究方向：动物胚胎工程。Tel: 025-84395381; E-mail: caeet@njau.edu.cn 
是作为组织工程中重要的种子细胞用于损伤和疾病 组织的修复和替代治疗研究, 同时在药理和毒理研 究方面也具有重要作用; 对于动物生产而言, 在核 移植、嵌合体、转基因动物研究方面也有着广阔的 应用前景。然而，一方面，目前真正建立ESC系的只 有小鼠 $[1,2]$ 、大鼠 $[3,4]$, 恒河猴 ${ }^{[5]}$ 和人 ${ }^{[6]}$, 其它动物还 没有真正的ESC系建立; 另一方面, 人类ESC研究一 直因为伦理学问题和免疫排斥问题的限制进展缓慢; 这些都表明通过传统方法建立ESC的局限性, 诱导 性多能干细胞(Induced pluripotent stem cells, iPSC) 技术的建立 ${ }^{[7]}$, 对解决这些问题提供了新的契机, 进一步深化和丰富了干细胞研究的内容。

\section{1 iPSC 技术}

iPSC 是将维持ESC多能性相关的一些核心基因 (Oct3/4, Sox2, Klf4, c-Myc, Nanog和Lin28等)转导入 非多能性的细胞中 (各种终末分化的体细胞或者成 体干细胞)后产生的类似多能性 ESC 的特殊细胞类 型。为了提高iPSC技术的效率及安全性, 目前主要 围绕筛选标记、载体系统、转导基因的种类、体细 胞类型和一些可以促进效率提高的化合物篮选等展 开研究。对于iPSC篮选标记, 从最初的Fbx15标记篮

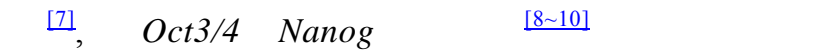
态学 ${ }^{[11]}$ 方法的篮选, 表明了诱导是一个渐进的过程, 保证诱导的时间很关键, 充分被诱导到多能状态从 而产生了可以种系传递的嵌合体小鼠; 对于载体系 统, 从最初的逆转录病毒 ${ }^{[7]}$ 到慢病毒 ${ }^{[12]}$, 再到非整 合的腺病毒 ${ }^{[13]}$, 从短暂表达的质粒载体 ${ }^{[14]}$, 到用 Cre/LoxP系统 ${ }^{[15]}$ 、oriP/EBNA1载体 ${ }^{[14]}$ 及piggyBac转 座系统 ${ }^{[16]}$ 等一类整合后可删除的基因表达系统，从 单一转导基因 ${ }^{[7]}$ 的单个转染到多个转导基因的共载 体 ${ }^{[17]}$ 转染, 整体上显示了从病毒载体到非病毒载体, 再到可删除的载体, 而且载体的载量也不断加大从 而减少载体的数量; 对于所转导的基因, 从最初建 立的Oct3/4、Sox2、Klf4、c-Myc 基因转导系统 ${ }^{[7]}$ 到 Oct3/4、Sox2、Nanog、Lin28基因转导系统 ${ }^{[12]}$, 从 Oct3/4、Sox2、Klf4、C-Myc、Nanog、Lin28六因子 系统 ${ }^{[18]}$ 再到后来的 3 个基因转导 ${ }^{[19] 、 2}$ 个基因转导 ${ }^{[20]}$ 和 1 个基因转导 [21], 总体上是转导基因种类不断减 少; 在体细胞类型的选择上, 从成纤维细胞 ${ }^{[7]}$ 到肝 细胞、胃上皮细胞 ${ }^{[22]}$ 、胰腺 $\beta$ 细胞 ${ }^{[23]}$ 、甚至成熟的B
淋巴细胞 ${ }^{[24]}$, 从胚胎阶段的细胞到生后期阶段的细 胞, 从干/祖细胞到终末分化阶段的细胞, 表明了各 种类型和不同分化阶段的细胞都具有重编程到多能 状态的潜能; 同时，为了进一步提高iPSC的产生效 率, 研究人员筛选了一系列小分子化合物, 比如, 组蛋白去乙酰化酶抑制剂2-丙基戊酸 (Valproic acid, $\mathrm{VPA})^{[25]}$ 、Wnt信号通路的重要蛋白 Wnt $3 a^{[26]}$ 、DNA

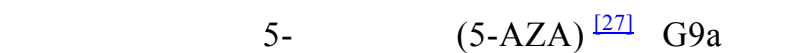
白甲基化转移酶抑制剂 (BIX01294) ${ }^{[28]}$ 、钲通道激动剂

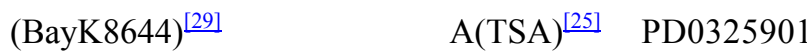

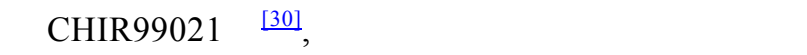
关重要的细胞信号通路和表观遗传修饰, 从而提高 诱导重编程的效率。目前最新的发展趋势是无载体 系统转导, 即通过转导基因的重组蛋白质直接处理 进行重编程 ${ }^{[31,32]}$ 。另外, RAN干扰(RNA i)技术同样 也可以通过干扰相关细胞信号通路和表观遗传修饰 来提高诱导重编程的效率。总体上, iPSC在不断朝着 简便、高效和安全的方向快速发展, 与ESC的研究方 向相同, iPSC的诱导分化及其可能的生物医学研究 也在不断拓展。

iPSC的产生证实了分化的细胞可以通过少数几 个因子的外源导入而被重编程到多能状态，为进一 步研究多能性的调控机制提供了新的研究模型, 这 种全新的体细胞重编程的方法在基础研究和临床应 用方面具有极其重大的意义。尽管制造iPSC的方法 近来不断优化，但目前对iPSC产生的具体分子机制 还不十分清楚，同时这种方法重编程的效率依然较 低，虽然已有一些动物实验初步显示了iPSC的临床 潜能，但是安全问题依然有待评估，所以iPSC应用 于临床还尚待时日, 尤其是要深化对iPSC产生机理 的研究。目前已经成功建立iPSC的物种有小鼠 ${ }^{[7]}$ 、 人 ${ }^{[12]}$ 、大鼠 ${ }^{[33]}$ 和猕猴 ${ }^{[34]}, 2009$ 开始陆续报道建立了 猪iPSC, 进一步拓展了iPSC技术的研究空间。

\section{2 猪 $\mathrm{PSC}$ 技术的建立}

猪由于其来源方便、结构和生理与人类的相似 及其在畜牧业中的重要地位等诸多优势，一直以来 就是有蹄类大动物 $\mathrm{ESC}$ 建系研究的重点, 然而由于 诸多因素的限制, 猪 $\mathrm{ESC}$ 系的建立一直进展缓慢。 iPSC技术开创了体细胞重编程的全新方法，所得 $\mathrm{iPSC}$ 具有和 ESC 相似的生物学特性, 不仅进一步解 
决了人类ESC研究面临的伦理学困境和免疫排斥问 题, 而且深化了对细胞多能性和基因组重编程的认 识 ${ }^{[35]}$ 。iPSC技术的另外一个优势就是对于还未成功 建立ESC系的物种而言, 通过建立iPSC有助于深入 了解其ESC的相关特性, 比如建立合适的培养系统、 $\mathrm{ESC}$ 的形态、生长特性、表面标记及多能性机制等。 近来猪iPSC的建立，一方面将有力促进猪 ESC系的 建立, 另一方面将拓展猪多能性干细胞在生物医学 和动物生产中的应用。

世界上首例报道建立猪iPSC系的是中国科学院 上海生命科学研究院生化与细胞生物学研究所肖否 博士实验室 ${ }^{[36]}$, 该实验室使用丹系长白猪的原代耳 皮肤成纤维细胞和原代骨髓细胞为供体细胞, 在证 明了猪细胞不表达逆转录病毒的受体后, 采用 Dox(强力霉素)诱导的慢病毒表达系统, 同时在EGFP 的下游插入四环素操纵子, 转入人源化的 6 个因子 (Oct3/4、Sox2、Klf4、C-Myc、Nanog和Lin28)2天后, 采用ESC培养基, 同时加入Dox开始诱导, 诱导 5 天 后开始出现克隆, 撤除Dox后继续培养6天后挑出克 隆进行鉴定, 包括形态学观察、AKP染色分析、端 粒酶分析、核型分析、基因芯片和定量PCR分析了 基因表达谱、亚硫酸氢盐测序检测表观遗传状态、 免疫细胞化学鉴定相关分子标记(SSEA3、SSEA4、

Tra-1-60、Tra-1-81、Oct3/4、Nanog、Sox2、Rex1和 $C D H 1)$ 等，体外分化和体内畸胎瘤分化实验表明可 以分化为 3 个胚层来源的细胞, 经过这些鉴定后表 明建立了猪iPSC, 然而研究没有进行嵌合体实验来 进一步验证猪iPSC的全能性。随后Esteban等 ${ }^{[37]}$ 分别 使用人源化和鼠源化的4因子(Oct3/4、Sox2、Klf4、 $c-M y c)$ 逆转录病毒诱导中国藏猪胎儿成纤维细胞成 iPSC。Ezashi等 ${ }^{[38]}$ 使用人源化的的4因子 (Oct3/4、

Sox2、KIf4、c-Myc)慢病毒转染系统建立了猪iPSC, 但是所建立的猪iPSC外源转导基因持续表达。最近 West等 ${ }^{[39]}$ 报道使用人源化的6因子(Oct3/4、Sox 2、

Klf4、c-Myc、Nanog和Lin28)诱导，建立了可以产生 成活嵌合体的猪iPSC, 这也是目前除了小鼠之外可 以产生成活嵌合体的iPSC, 表明猪iPSC全能性得到 了更严格的鉴定。目前关于猪iPSC研究报道还十分 有限，但进展迅速，在以上各种鉴定方法的基础上, 如果能够进行最终的四倍体囊胚补偿实验获得猪 iPSC克隆动物, 这将最终证明所获得的猪iPSC为真
正的多能性干细胞, 相信随着猪iPSC技术的完善, 由iPSC产生的克隆猪将会诞生。

\section{3 猪 iPSC 技术的研究价值}

猪 $\mathrm{PSCC}$ 的建立具有重大的研究价值, 不仅提供 了一种研究有蹄类大动物 iPSC 的模型, 同时在生物 医学和动物生产中也具有潜在的前景, 猪 PSC 提供 的信息将促进猪 $\mathrm{ESC}$ 系的建立，对其它家畜包括 牛、绵羊、山羊等多能干细胞的建系也具有一定的 借鉴意义。

\section{1 人类组织器官工程}

目前世界范围内器官移植面临的一个最大问题 就是供体器官的短缺, 而且器官需求一直在增加, 供求矛盾十分尖锐, 而猪在组织器官的大小、结构 和功能方面与人类极为相似, 同时人猪之间疾病转 移的概率较低, 使得猪成为了人类器官移植最佳异 种来源的供体动物。多年来, 猪来源的胰岛素、心 脏瓣膜和皮肤在人类糖尿病治疗和组织器官移植临 床的应用充分证明了可行性 ${ }^{[40]}$ 。然而异种移植的强 烈的免疫排斥反应一直尚未解决 ${ }^{[41]}$ 。最近有研究报 道对非人灵长类猕猴建立糖尿病模型后, 移植猪妊 娠特定阶段胎儿胰腺组织可以成功治疗糖尿病 ${ }^{[42]}$, 但是长期的慢性免疫排斥反应仍然是个问题。敲除 $\alpha$-1，3-半乳糖苷转移酶基因被证明是一种有效的克 服免疫排斥反应的方法 ${ }^{[43]}$ 。然而传统基因敲除技术 因为猪ESC尚未建立而效率低下。猪iPSC建系后, 结 合克隆技术和基因打靶技术，可以高效生产可用于 人类移植的猪源化组织器官, 避免了由于猪内皮细 胞表面的糖分子容易和人类抗体结合从而引发强烈 的免疫反应, 建立和人体免疫系统相容的组织器官, 从而缓解目前人类器官移植面临的供体严重不足和 免疫排斥的问题。

\section{2 人类医学研究模型}

相对于其它动物来讲, 猪和人类在解剖、生理、 心血管系统、消化系统、皮肤结构和营养需要上非 常相似, 使得猪成为了生物医学研究中一种更好的 动物模型 ${ }^{[44]}$, 尤其是在一些研究领域具有重要的价 值, 比如癌症、心血管疾病、糖尿病、血液疾病、 烧伤病、器官移植、营养代谢病以及药物安全性评 价方面, 加深我们对于人类功能正常和病理条件下 
机制的理解。目前构建的猪动脉粥样硬化和糖尿病 模型已经成功的应用于医学动物实验中 ${ }^{[45]}$ 。另外, 我国一些特有的小型猪正在进行实验动物化研究, 其主要品种有版纳微型猪近交系、五指山小型猪近 交系、广西巴马小型猪、贵州小型香猪、甘肃蕨麻 小型猪、藏猪等, 这些小型猪iPSC的建系, 可以用来 研究基因功能、细胞分化和个体发育方面的一些基 础科学问题, 还可以用来研究人类疾病的机制, 相 对于小鼠细胞实验具有更大程度的可靠性。由于猪 的基因组与人类高度同源 ${ }^{[46]}$, 许多人类的单基因变 异导致的遗传疾病, 都可以对猪iPSC进行基因修饰 后建立同样基因改变的猪疾病模型, 可以在细胞和 动物整体水平上研究疾病的致病机理, 从而开发治 疗该疾病的方法, 所得出的结果比小鼠和大鼠模型 更接近人类, 更具有应用价值。

\section{3 为建立家畜 ESC 奠定基础}

猪的iPSC系建立之前, 人们曾建立过许多猪的 类ESC系 ${ }^{[47 \sim 49]}$, 但是却一直对猪 $\mathrm{ESC}$ 的形态、分子标 记等方面认识存在争议, 而猪iPSC系建立后可以给 予一些有关猪ESC的信息, 比如目前建立的猪iPSC 药物诱导系统 ${ }^{[35]}$ 提供了一个研究鉴定猪多能性干细 胞自我更新的小分子化合物和生长因子的技术平台, 这将有助于猪ESC系的建立。另外, 也可以利用iPSC 技术来建立其他物种, 如牛、羊等的多能干细胞系, 掌握这些多能干细胞系所具有的各种干细胞特性, 研究其保持全能性的内在信号通路或外在影响因子, 这些信息将有利于最终从这些物种的囊胚中获得真 正的ESC系, 而且家畜iPSC系若能建立将会有力促 进家畜相关胚胎生物技术的发展。

\section{4 实现高效的基因修饰, 为基因打靶提供供体细胞}

自从 1997 年第一只体细胞克隆绵羊诞生以来 ${ }^{[50]}$, 迄今至少有包括猪在内的 13 种哺乳动物被成功克 隆, 而在大型哺乳动物中, 猪的核移植难度最大。 2000 年获得世界首例体细胞核移植猪后 ${ }^{[51]}$, 世界范 围内又有多个基因修饰猪相继出生, 然而猪转基因 克隆一直面临着核移植效率低下、基因的随机整合 和整合率低下等瓶颈问题, 而ESC相对于体细胞拥 有更高的核移植效率，同时ESC结合同源重组技术 可以进一步提高所转基因的整合效率而且能够实现 定点整合, 但是猪 $\mathrm{ESC}$ 系却一直未能真正建立。目前
猪iPSC系的建立重新点燃了人们的希望, iPSC具有 类似于ESC的增殖特性, 能够在适当的培养条件下 长期保持增殖而不发生分化, 将可能为我们开展基 因打靶提供足够的细胞和充足的时间，篮选到目的 细胞后, 可以大批量持续地提供同质的供体核, 促 进转基因动物的生产。通过整合集成猪iPSC技术、 同源重组技术和克隆技术，为高效生产基因修饰猪 开辟了一条新的技术路线。

\section{5 保种和育种}

作为一个世界养猪大国, 我国拥有世界上最丰 富的地方猪品种资源, 然而为了适应市场需求, 多 年来大量引进国外品种进行大规模的杂交利用, 这 些种质资源的引进一方面促进了中国养猪业的发展, 另一方面也带来了对地方品种资源的冲击，一些珍 稀地方猪种遗传资源亟须保护。猪iPSC的建系提供 一种新的保种素材, 可以通过液氮冷冻达到长期保 存。有研究表明, 人iPSC可以在体外分化为PGC, 从 而为人iPSC进一步分化为精子或卵子提供可能 ${ }^{[52]}$ 。同 样, 猪iPSC如果具有充分的多能性, 就可以在体外 诱导其定向分化为精子或者卵子, 进一步丰富保种 的手段和内容。对于育种, 通过对猪iPSC进行基因 打靶定点整合一些可以提高猪生长速度或增强抗病 能力的基因, 或者定点敲除一些疾病易感基因生产 具有特定性状的基因修饰猪, 从而达到高效定向育 种的目的。

\section{4 展望}

随着iPSC技术朝着安全、高效和简便的方向不 断发展, 猪iPSC技术也将不断完善, 一方面, 通过 优化获取猪iPSC的技术方案进一步提高猪iPSC技术 的效率和安全性, 并且深入研究猪体细胞表观重编 程的内在分子机制; 另一方面, 对建立的猪iPSC和 类ESC进行对比研究, 深入揭示二者在形态、增殖特 性以及细胞表面特异性标志方面的异同, 通过猪 $\mathrm{iPSC}$ 提供的丰富信息加深对猪ESC的理解并最终建 立真正的猪 $\mathrm{ESC}$ 系。尽管目前能够参与种系传递的 嵌合体猪已经诞生, 但是仍然需要通过更严格的实 验进一步检验所获得猪iPSC的全能性和多能性, 包 括通过四倍体囊胚补偿法生产来源于猪iPSC的克隆 猪。另外, 还需要建立猪iPSC诱导分化研究的技术 
平台, 诱导猪iPSC向各种重要的终末细胞分化, 提 供人类疾病研究的猪细胞实验模型，同时也为人类 各种疾病的细胞治疗提供新的来源。未来随着猪 $\mathrm{iPSC}$ 克隆猪的出生, 通过猪iPSC技术生产的各种转 基因克隆猪将陆续诞生，这不仅会促进猪产业高新 技术的发展，更会在广阔的生物医学领域中发挥深 远的影响。

\section{参考文献(References):}

[1] Evans MJ, Kaufman MH. Establishment in culture of pluripotential cells from mouse embryos. Nature, 1981, 292(5819): 154-156.[DOI]

[2] Martin GR. Isolation of a pluripotent cell line from early mouse embryos cultured in medium conditioned by teratocarcinoma stem cells. Proc Natl Acad Sci USA, 1981, 78(12): 7634-7638. [DOI]

[3] Buehr M, Meek S, Blair K, Yang J, Ure J, Silva J, McLay R, Hall J, Ying QL, Smith A. Capture of authentic embryonic stem cells from rat blastocysts. Cell, 2008, 135(7): 1287-1298. [DOI]

[4] Li P, Tong C, Mehrian-Shai R, Jia L, Wu N, Yan YZ, Maxson RE, Schulze EN, Song HY, Hsieh CL, Pera MF, Ying QL. Germline competent embryonic stem cells derived from rat blastocysts. Cell, 2008, 135(7): 1299-1310. [DOI]

[5] Thomson JA, Kalishman J, Golos TG, Durning M, Harris CP, Becker RA, Hearn JP. Isolation of a primate embryonic stem cell line. Proc Natl Acad Sci USA, 1995, 92(17): 7844-7848. [DOI]

[6] Thomson JA, Itskovitz-Eldor J, Shapiro SS, Waknitz MA, Swiergiel JJ, Marshall VS, Jones JM. Embryonic stem cell lines derived from human blastocysts. Science, 1998, 282(5391): 1145-1147[DOI]

[7] Takahashi K, Yamanaka S. Induction of pluripotent stem cells from mouse embryonic and adult fibroblast cultures by defined factors. Cell, 2006, 126(4):663-676. [DOI]

[8] Okita K, Ichisaka T, Yamanaka S. Generation of germline-competent induced pluripotent stem cells. Nature, 2007, 448(7151): 313-317. [DOI]

[9] Maherali N, Sridharan R, Xie W, Utikal J, Eminli S, Arnold K, Stadtfeld M, Yachechko R, Tchieu J, Jaenisch R, Plath K, Hochedlinger K. Directly reprogrammed fibroblasts show global epigenetic remodeling and widespread tissue contribution. Cell Stem Cell, 2007, 1(1): 55-70. [DOI]

[10] Wernig M, Meissner A, Foreman R, Brambrink T, Ku M,
Hochedlinger K, Bernstein BE, Jaenisch R. In vitro reprogramming of fibroblasts into a pluripotent ES-cell-like state. Nature, 2007, 448(7151): 318-324. [DOI]

[11] Meissner A, Wernig M, Jaenisch R. Direct reprogramming of genetically unmodified fibroblasts into pluripotent stem cells. Nat Biotechnol, 2007, 25(10): 1177-1181. [DOI]

[12] Yu JY, Vodyanik MA, Smuga-Otto K, Antosiewicz-Bourget J, Frane JL, Tian SL, Nie J, Jonsdottir GA, Ruotti V, Stewart R, Slukvin II, Thomson JA. Induced pluripotent stem cell lines derived from human somatic cells. Science, 2007, 318(5858): 1917-1920. [DOI]

[13] Stadtfeld M, Nagaya M, Utikal J, Weir G, Hochedlinger K. Induced pluripotent stem cells generated without viral integration. Science, 2008, 322(5903): 945-949. [DOI]

[14] Yu JY, Hu KJ, Smuga-Otto K, Tian SL, Stewart R, Slukvin II, Thomson JA. Human induced pluripotent stem cells free of vector and transgene sequences. Science, 2009, 324(5928): 797-801. [DOI]

[15] Kaji K, Norrby K, Paca A, Mileikovsky M, Mohseni P, Woltjen K. Virus-free induction of pluripotency and subsequent excision of reprogramming factors. Nature, 2009 , 458(7239): 771-775. [DOI]

[16] Woltjen K, Michael IP, Mohseni P, Desai R, Mileikovsky M, Hämäläinen R, Cowling R, Wang W, Liu PT, Gertsenstein M, Kaji K, Sung HK, Nagy A. piggyBac transposition reprograms fibroblasts to induced pluripotent stem cells. Nature, 2009, 458(7239): 766-770. [DOI]

[17] Okita K, Nakagawa M, Hyenjong H, Ichisaka T, Yamanaka S. Generation of mouse induced pluripotent stem cells without viral vectors. Science, 2008, 322(5903): 949-953[DOI]

[18] Liao J, Wu Z, Wang Y, Cheng L, Cui C, Gao Y, Chen TT, Rao LJ, Chen SY, Jia NN, Dai HM, Xin SM, Kang JH, Pei G, Xiao L. Enhanced efficiency of generating induced pluripotent stem (iPS) cells from human somatic cells by a combination of six transcription factors. Cell Research, 2008, 18(5): 600-603. [DOI]

[19] Nakagawa M, Koyanagi M, Tanabe K, Takahashi K, Ichisaka T, Aoi T, Okita K, Mochiduki Y, Takizawa N, Yamanaka S. Generation of induced pluripotent stem cells without Myc from mouse and human fibroblasts. Nat Biotechnol, 2008, 26(1): 101-106[DOI]

[20] Kim JB, Zaehres H, Wu GM, Gentile L, Ko K, Sebastiano V, Araúzo-Bravo MJ, Ruau D, Han DW, Zenke M, Schöler HR. Pluripotent stem cells induced from adult neural stem cells by reprogramming with two factors. Nature, 2008, 454(7204): 646-650. [DOI]

[21] Kim JB, Sebastiano V, Wu GM, Araúzo-Bravo MJ, Sasse P, 
Gentile L, Ko K, Ruau D, Ehrich M, van den Boom D, Meyer J, Hübner K, Bernemann C, Ortmeier C, Zenke M, Fleischmann BK, Zaehres H, Schöler HR. Oct4-induced pluripotency in adult neural stem cells. Cell, 2009, 136(3): 411-419. [DOI]

[22] Aoi T, Yae K, Nakagawa M, Ichisaka T, Okita K, Takahashi K, Chiba T, Yamanaka S. Generation of pluripotent stem cells from adult mouse liver and stomach cells. Science, 2008, 321(5889): 699-702. [DOI]

[23] Stadtfeld M, Brennand K, Hochedlinger K. Reprogramming of pancreatic $\beta$ cells into induced pluripotent stem cells. Curr Biol, 2008, 18(12): 890-894. [DOI]

[24] Hanna J, Markoulaki S, Schorderet P, Carey BW, Beard C, Wernig M, Creyghton MP, Steine EJ, Cassady JP, Foreman R, Lengner CJ, Dausman JA, Jaenisch R. Direct reprogramming of terminally differentiated mature B lymphocytes to pluripotency. Cell, 2008, 133(2): 250-264. [DOI]

[25] Huangfu D, Maehr R, Guo WJ, Eijkelenboom A, Snitow M, Chen AE, Melton DA. Induction of pluripotent stem cells by defined factors is greatly improved by small-molecule compounds. Nat Biotech, 2008, 26(7): 795-797. [DOI]

[26] Marson A, Foreman R, Chevalier B, Bilodeau S, Kahn M, Young RA, Jaenisch R. Wnt signaling promotes reprogramming of somatic cells to pluripotency. Cell Stem Cell, 2008, 3(2): 132-135. [DOI]

[27] Mikkelsen T S, Hanna J, Zhang XL, Ku M, Wernig M, Schorderet P, Bernstein BE, Jaenisch R, Lander ES, Meissner A. Dissecting direct reprogramming through integrative genomic analysis. Nature, 2008, 454(7200): 49-55. [DOI]

[28] Shi Y, Do JT, Desponts C, Hahm HS, Schöler HR, Ding S. A combined chemical and genetic approach for the generation of induced pluripotent stem cells. Cell Stem Cell, 2008, 2(6): 525-528. [DOI]

[29] Shi Y, Desponts C, Do JT, Hahm HS, Schöler HR, Ding S. Induction of pluripotent stem cells from mouse embryonic fibroblasts by Oct4 and Klf4 with small-molecule compounds. Cell Stem Cell, 2008, 3(5): 568-574. [DOI]

[30] Silva J, Barrandon O, Nichols J, Kawaguchi J, Theunissen TW, Smith A. Promotion of reprogramming to ground state pluripotency by signal inhibition. PLOS Biol, 2008, 6(10): e253. [DOI]

[31] Zhou HY, Wu SL, Joo JY, Zhu SY, Han DW, Lin TX, Trauger S, Bien G, Yao SS, Zhu Y, Siuzdak G, Schöler HR, Duan LX, Ding S. Generation of induced pluripotent stem cells using recombinant proteins. Cell Stem Cell, 2009, 4(5): 381-384. [DOI]

[32] Kim D, Kim CH, Moon JI, Chung YG, Chang MY, Han BS, Ko S, Yang E, Cha KY, Lanza R, Kim KS. Generation of hu- man induced pluripotent stem cells by direct delivery of reprogramming proteins. Cell Stem Cell, 2009, 4(6): 472-476. [DOI]

[33] Liao J, Cui C, Chen SY, Ren JT, Chen JJ, Gao Y, Li H, Jia NN, Cheng L, Xiao HS, Xiao L. Generation of induced pluripotent stem cell lines from adult rat cells. Cell Stem Cell, 2009, 4(1): 11-15. [DOI]

[34] Liu HS, Zhu FF, Yong J, Zhang PB, Hou PP, Li HG, Jiang W, Cai J, Liu M, Cui K, Qu XX, Xiang TT, Lu DY, Chi XC, Gao G, Ji WZ, Ding MX, Deng HK. Generation of induced pluripotent stem cells from adult rhesus monkey fibroblasts. Cell Stem Cell, 2008, 3(6): 587-590. [DOI]

[35] Kang L, Kou ZH, Zhang Y, Gao SR. Induced pluripotent stem cells (iPSCs) - a new era of reprogramming. $J$ Genet Genomics, 2010, 37(7): 415-421. [DOI]

[36] Wu Z, Chen JJ, Ren JT, Bao L, Liao J, Cui C, Rao LJ, Li H, Gu YJ, Dai HM, Zhu H, Teng XK, Cheng L, Xiao L. Generation of pig induced pluripotent stem cells with a drug-inducible system. J Mol Cell Biol, 2009, 1(1): 46-54.[DOI]

[37] Esteban MA, Xu JY, Yang JY, Peng MX, Qin DJ, Li W, Jiang ZX, Chen JK, Deng K, Zhong M, Cai JL, Lai LX, Pei DQ. Generation of induced pluripotent stem cell lines from Tibetan miniature pig. J Biol Chem, 2009, 284(26): 17634-17640. [DOI]

[38] Ezashi T, Telugu BP, Alexenko AP, Sachdev S, Sinha S, Roberts RM. Derivation of induced pluripotent stem cells from pig somatic cells. Proc Natl Acad Sci USA, 2009, 106(27): 10993-10998. [DOI]

[39] West FD, Terlouw SL, Kwon DJ, Mumaw JL, Dhara SK, Hasneen K, Dobrinsky JR, Stice SL. Porcine induced pluripotent stem cells produce chimeric offspring. Stem Cells Dev, 2010, 19(8): 1211-1220. [DOI]

[40] Hall V. Porcine embryonic stem cells: a possible source for cell replacement therapy. Stem Cell Rev, 2008, 4(4): 275-282. [DOI]

[41] Cooper DK. Clinical xenotransplantion-how close are we? Lancet, 2003, 362(9383): 557-559. [DOI]

[42] Hecht G, Eventov-Friedman S, Rosen C, Shezen E, Tchorsh D, Aronovich A, Freud E, Golan H, El-Hasid R, Katchman H, Hering BJ, Zung A, Kra-Oz Z, Shaked-Mishan P, Yusim A, Shtabsky A, Idelevitch P, Tobar A, Harmelin A, Bachar-Lustig E, Reisner Y.. Embryonic pig pancreatic tissue for the treatment of diabetes in a nonhuman primate model. Proc Natl Acad Sci USA, 2009, 106(21): 8659-8664. [DOI]

[43] Kolber-Simonds D, Lai LX, Watt SR, Denaro M, Arn S, Augenstein ML, Betthauser J, Carter DB, Greenstein JL, Hao YH, Im GS, Liu ZH, Mell GD, Murphy CN, Park KW, Rieke 
A, Ryan DJJ, Sachs DH, Forsberg EJ, Prather RS, Hawley RJ. Production of $\alpha$-1, 3-galactosyltransferase null pigs by means of nuclear transfer with fibroblasts bearing loss of heterozygosity mutations. Proc Natl Acad Sci USA, 2004, 101(19): 7335-7340. [DOI]

[44] Schook L, Beattie C, Beever J, Donovan S, Jamison R, Zuckermann F, Niemi S, Rothschild M, Rutherford M, Smith D. Swine in biomedical research: creating the building blocks of animal models. Anim Biotechnol, 2005, 16(2): 183-190. [DOI]

[45] Turk JR, Henderson KK, Vanvickle GD, Watkins J, Laughlin MH. Arterial endothelial function in a porcine model of early stage atherosclerotic vascular disease. Int J Exp Pathol, 2005, 86(5): 335-345. [DOI]

[46] Lunney JK. Advances in swine biomedical model genomics. Int J Biol Sci, 2007, 3(3): 179-184. [DOI]

[47] Notarianni E, Laurie S, Moor RM, Evans MJ. Maintenance and differentiation in culture of pluripotential embryonic cell lines from pig blastocysts. J Reprod Fertil Suppl, 1990, 41: 51-56. [DOI]

[48] Strojek RM, Reed MA, Hoover JL, Wagner TE. A method for cultivating morphologically undifferentiated embryonic stem cells from porcine blastocysts. Theriogenology, 1990, 33(4): 901-913. [DOI]

[49] Piedrahita JA, Anderson GB, Bondurant RH. On the isolation of embryonic stem cells: comparative behavior of murine, porcine and ovine embryos. Theriogenology, 1990, 34(5): 879-901. [DOI]

[50] Wilmut I, Schnieke AE, McWhir J, Kind AJ, Campbell KHS. Viable offspring derived from fetal and adult mammalian cells. Nature, 1997, 385(6619): 810-813. [DOI]

[51] Polejaeva IA, Chen SH, Vaught TD, Page RL, Mullins J, Ball S, Dai YF, Boone J, Walker S, Ayares DL, Colman A, Campbell KHS. Cloned pigs produced by nuclear transfer from adult somatic cells. Nature, 2000, 407(6800): 86-90. [DOI]

[52] Park TS, Galic Z, Conway AE, Lindgren A, van Handel BJ, Magnusson M, Richter L, Teitell MA, Mikkola HK, Lowry WE, Plath K, Clark AT. Derivation of primordial germ cells from human embryonic and induced pluripotent stem cells is significantly improved by coculture with human fetal gonadal cells. Stem Cells, 2009, 27(4): 783-795. [DOI]

\section{・科学新闻・}

\section{水稻抗病防御反应分子机制研究取得重要进展}

中国科学院遗传与发育生物学研究所植物基因组学国家重点实验室储成才课题组和中国水稻所水稻生物学国家重 点实验室朱旭东课题组合作, 通过对不同水稻突变体库进行大规模篮选, 获得两个不完全显性、叶鞘特异性自主坏死的 突变体 nls1-1D 和 nls1-2D。这种自主性坏死斑主要以发育依赖方式出现在叶鞘上, 类似于植物抗病防御反应过程中出 现的超敏反应(hypersensitive response)。通过图位克隆方法，储成才课题组的唐九友博士鉴定到相应的基因 NLS1，该基 因编码典型的 CC-NB-LRR 类 R 蛋白。转基因互补和 RNA 干扰实验进一步确认 NLS1 的功能获得性突变导致了突变体 特定组织细胞的自主性死亡。与突变体坏死斑形成的模式相一致, NLS1 基因在叶鞘上表达最高, 且这种表达同样具有发 育依赖性。另一方面，在没有出现坏死斑的其他组织同样检测到 NLS1 相对低水平的表达，表明 NLS1 可能在特定组织 激活特定的抗病防御信号途径从而导致细胞死亡。

nls1-1D 和 nls1-2D 的突变均是 CC-NB-LRR 类 R 蛋白 NLS1 的 NB 结构域上单个氨基酸的替换。随着 NLS1 这一 $\mathrm{R}$ 蛋白的序列改变, nls1 突变体内积累了高水平的水杨酸(SA, salicylic acid), 组成性诱导了抗病基因的表达, 同时提高 了对水稻白叶枯病菌(Xoo)的抗性。与此同时, 尽管 nls1-1D 突变体上坏死斑的表型与其体内 NLS1 转录本的大量积累有 着一定的相关性，但是过表达野生型 NLS1 基因并不能诱导自主性坏死斑的形成和抗病相关基因 $P R 1 b$ 的表达，且没有 改变对 Xoo 病原菌的抗性, 表明突变体的表型并非简单的由 NLS1 的过量表达所引起。因此, nls1-1D 和 nls1-2D 的突 变所引起的单个氨基酸替换导致了 NLS1 蛋白自主性的激活。

为了探讨 nls1-1D 植株中 SA 的高水平积累是否是下游抗病防御途径激活的必需步骤，唐九友博士等通过在野生型 和 nls1-1D 突变体中表达 SA 水解酶基因 NahG, 从而获得相应的 SA 缺失的转基因植株, 有趣的是, 对这些转基因植株 的分析表明, NLS1 介导的抗病防御反应并未受到 SA 的含量和 NPR1 表达下调的影响。从而表明, NLS1 可不依赖于 SA 和 NPR1 信号传递途经激活下游抗病防御反应，这一发现揭示了水稻 $\mathrm{R}$ 基因介导的抗病防御反应的新机制。该成果已 于 2011 年 3 月 21 日在国际杂志 Plant Journal 在线发表(DOI: 10.1111/j.1365-313X.2011.04557.x), 该研究得到了科技部 和国家自然科学基金委的资助。 